\title{
AUTOMOBILE SPEED DETECTION AND COLLISION AVOIDANCE RADAR (ASDCAR)
}

\author{
P. PydiSaiCharan ${ }^{1}$, Ambati Nikhil ${ }^{2}$, ItlaSnehaMounika ${ }^{3}$, V. ShyamSandeep ${ }^{4}$, GantaHaswanth \\ Kumar $^{5}$, MahboobBaig ${ }^{6}$, B. Sada Siva ${ }^{7}$ \\ ${ }^{I}$ Student of Department of Electronics and Communication Engineering GayatriVidyaParishad College for Degree \\ and PG Courses School of Engineering Visakhapatnam, India \\ ${ }^{2}$ Student of Department of Electronics and Communication Engineering GayatriVidyaParishad College for Degree \\ and PG Courses School of Engineering Visakhapatnam, India \\ ${ }^{3}$ Student of Department of Electronics and Communication Engineering GayatriVidyaParishad College for Degree \\ and PG Courses School of Engineering Visakhapatnam, India \\ ${ }^{4}$ Student of Department of Electronics and Communication Engineering GayatriVidyaParishad College for Degree \\ and PG Courses School of Engineering Visakhapatnam, India \\ ${ }^{5}$ Student of Department of Electronics and Communication Engineering GayatriVidyaParishad College for Degree \\ and PG Courses School of Engineering Visakhapatnam, India \\ ${ }^{6}$ Student of Department of Electronics and Communication Engineering GayatriVidyaParishad College for Degree \\ and PG Courses School of Engineering Visakhapatnam, India \\ ${ }^{7}$ Under the Guidance of Professor and Head of the Department of Electronics and Communication Engineering \\ GayatriVidyaParishad College for Degree and PG Courses School of Engineering, Visakhapatnam, India.
}

\begin{abstract}
The most unwanted thing to happen to a road user is road accident. One serious road accident in the country occurs every minute and 16 die on Indian roads every hour.Most of the fatal accidents occur due to over speeding. Faster vehicles are more prone to accident than the slower one. Among the various methods for detecting speed of the vehicle, object detection systems based onRadar have been replaced for about a century for various purposes like detection of aircrafts, spacecraft, ships, navigation, reading weather formations and terrain mapping. The essential feature in adaptive vehicle activated sign systems is the accurate measurement of a vehicle's velocity; this measured velocity is used to take the necessary remedies to avoid accidents.
\end{abstract}

Keywords: -Doppler Effect, Doppler frequency shift, Automobile Speed Detection and Collision Avoidance Radar $(A S D C A R)$

\section{INTRODUCTION}

This project major focus will be on the making the vehicle driving safer. The radar used in vehicle Speed detection is called a Doppler radar.It is specialized radar that uses the Doppler Effect to producevelocity data about objects at a distance. It does this by bouncing a microwave signal off a desired targetand analyzing how the object's motion has altered the frequency of the returned signal.This variation gives direct and highly accurate measurements ofthe radial component of a target's velocity relative to the radar.Doppler radars are also used for aviation, sounding Satellites, meteorology, radar guns, radiology and healthcare.

In the conventional Doppler radars the source is stationary only the target is in the motion. But in the Automobile Speed Detection and Collision Avoidance Radar both source and target are in motion. We know that the Doppler Effect is the same as that for reflection of light by a moving mirror.The Doppler Effect causes the received frequency of a source to differ from the sent frequency if there is motion that is change in the distance between the source and the target.

The Doppler Effect is also known as Doppler FrequencyShift which is given by the equation in the case of source and the target both are in motion:

$$
f s=f o\left(\frac{c+v}{c-v}\right)
$$

Where ' $c$ ' as the speed of light, ' $v$ ' is the velocity of the target, ' $f s$ ' is the shifted frequency and ' $f_{o}$ ' is the original frequency.

Doppler frequency ' $f_{d}$ ' is given by the equation: $f d=f s-$ $f o=2 v \frac{f o}{(c-v)}$

Since most practical applications $\mathrm{v}<<\mathrm{c}$, then the value of $(\mathrm{c}$ - v) $\rightarrow \mathrm{c}$ then, $f d \cong 2 v \frac{f o}{c}$ 


\section{AUTOMOBILE SPEED DETECTION AND}

\section{COLLISION AVOIDER RADAR}

Automobile Speed Detection and Collision Avoider Radar System is capable to perform the task of primary radar whichis a long range radar $(40-60 \mathrm{~m})$ and secondary radar which is a short range radar $(6-10 \mathrm{~m})$ and a Microwave Radar Human Body Induction SwitchModule (5 - 10 $\mathrm{m})$. These two radars are Doppler radars function based on Doppler Effect. These are placed at front and rear ends of the vehicle, the data from radar is collected and displayed. Then it is analyzed, if the degree of closeness between the vehicles is increased then the necessary action of applying the brakes to reduce the speed is imitated automatically to avoid collision.

The design of the radardeal with HB100 Doppler Speed Sensor of $10.525 \mathrm{GHzfrequency}$ and RCWL-0516 Microwave Radar Sensor Module Human Body Induction Switch Module Intelligent Sensor for the development of the short range $(5-10 \mathrm{~m})$ prototype of Automobile Speed Detection and Collision Avoidance Radar.

The design of the Automobile Speed Detection and Collision Avoidance Radar with the primary and secondary radar and Human body induction switch module is developed with the operating frequency of $23.5 \mathrm{GHz}$ range the antenna used in this radar module is the rectangular patch micro strip patch antenna simulated in ANSYS HFSS 15.0. The function of the radar modules is developed in NI LAB View 2014 by the simulation process the radar system operation is tested and verified. The model of Automobile Speed Detection and Collision Avoidance Radar is simulated and tested and its working prototype is in process. The ASDCAR short range prototype is designed and it is functional. The detail description of the radar short range prototype and also the simulation results of the Automobile Speed Detection and Collision Avoidance Radar with 23.5 $\mathrm{GHz}$ frequencyare mentioned in this research paper.

\subsection{Block Diagram}

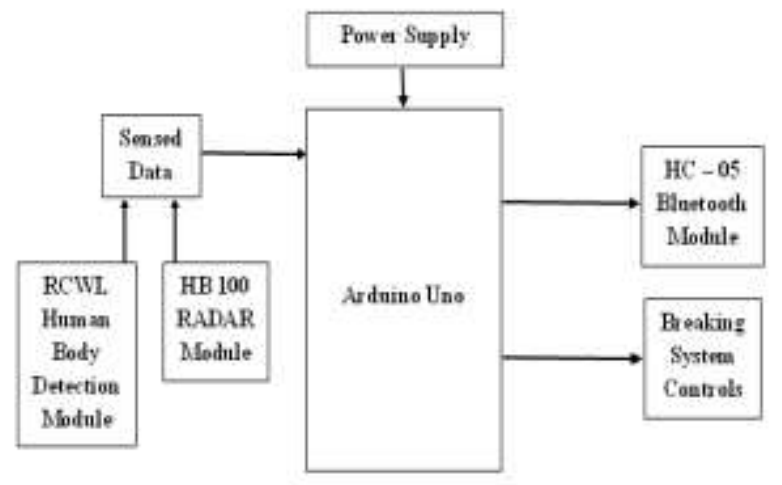

Fig 1: Internal Block Diagram of the Automobile Speed Detection and Collision Avoidance Radar
The block diagram of the Automobile Speed Detection and Collision Avoidance Radar consist of two main sensors HB 100 Radar module and RCWL - 0516 Human body induction switch module. The data sensed from these modules is provided to the digital data inputs of the ArduinoUno Controller board.

Sensor data is then analyzed if the vehicle is over speeding and degree of closeness between the two vehicles is increased the automatically the vehicle speed is reduced by providing the data to the breaking system controls which are connected to the digital output ports of the Arduino Uno.

In this prototype of radar we are using a Bluetooth Controlled Vehicle its operation is based on theclockwise and anti-clockwise movement of the motor which is connected to the Arduino Uno and data is transferred to the controlling device using the $\mathrm{HC}-05$ Bluetooth Module and the controlling device used is a mobile phone

Mobile application has been developed which is used to communicate with the Arduino Uno with the help of Bluetooth module. By using this application we can control the vehicle and this application also displays the speed of the vehicle.

\section{RADAR MODULES}

\subsection{HB 100 Radar Module}

HB100 radar module can detect the distance within $20 \mathrm{~m}$ and its transmitted frequency is $10.525 \mathrm{GHz}$.

It consists of Dielectric Resonator Oscillator (DRO), microwave mixer and path antenna. The oscillator is used to produce a sinusoidal wave of frequency $10.525 \mathrm{GHz}$, patch antenna connected to the oscillator radiates the wave towards the target and the reflected wave is received by another set of patch antenna.

The mixer mixes these two signals to generate a sinusoidal wave with frequency equal to the difference between the two signals. Figure 2 below shows the internal structure of HB100.

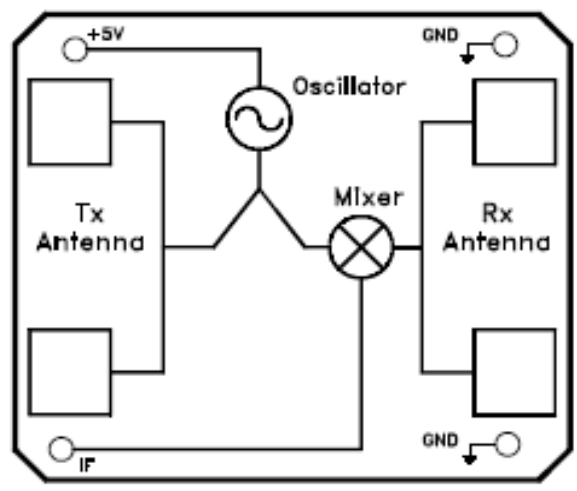

Fig 2: Internal Structure of the HB 100 
Power supply:HB100 powered continuous DC power supply $(\mathrm{CW})$ mode, and pulsation power supply (PW) mode of the two: HB100 adapt to the voltage range of $5 \mathrm{~V} \pm 5 \%$. Continuous DC power supply (CW) mode with a typical current of $35 \mathrm{~mA}$. Low duty cycle pulse power supply (PW) mode, 3 to $10 \%$ duty cycle pulse supply an average current of $1.2 \mathrm{~mA}$ to $4 \mathrm{~mA}$.

RF output: In any of the two recommended operating mode, HB100 is very low, both in constitute any hazard to the human body within the safe range. Continuous DC power supply $(\mathrm{CW})$ mode, with a total output power of less than $15 \mathrm{~mW}$. Output power density in the $5 \mathrm{~mm}$ at $1 \mathrm{~mW} / \mathrm{cm}^{2}$, $1 \mathrm{~m}$ at $0.72 \mathrm{~W} / \mathrm{cm}^{2}$. When the at a $5 \%$ duty cycle of the pulse powered mode, the power density were reduced to the level of $50 \mathrm{~W} / \mathrm{cm}^{2}$, and $0.036 \mathrm{~W} / \mathrm{cm}^{2}$.

IF Output: When the object in HB100 $1 \mathrm{~m} / \mathrm{s}$ with respect HB100 antenna surface, HB100 72Hz / s IF pulsation of the output frequency with the object relative radial movement speed is approximately linear relationship.

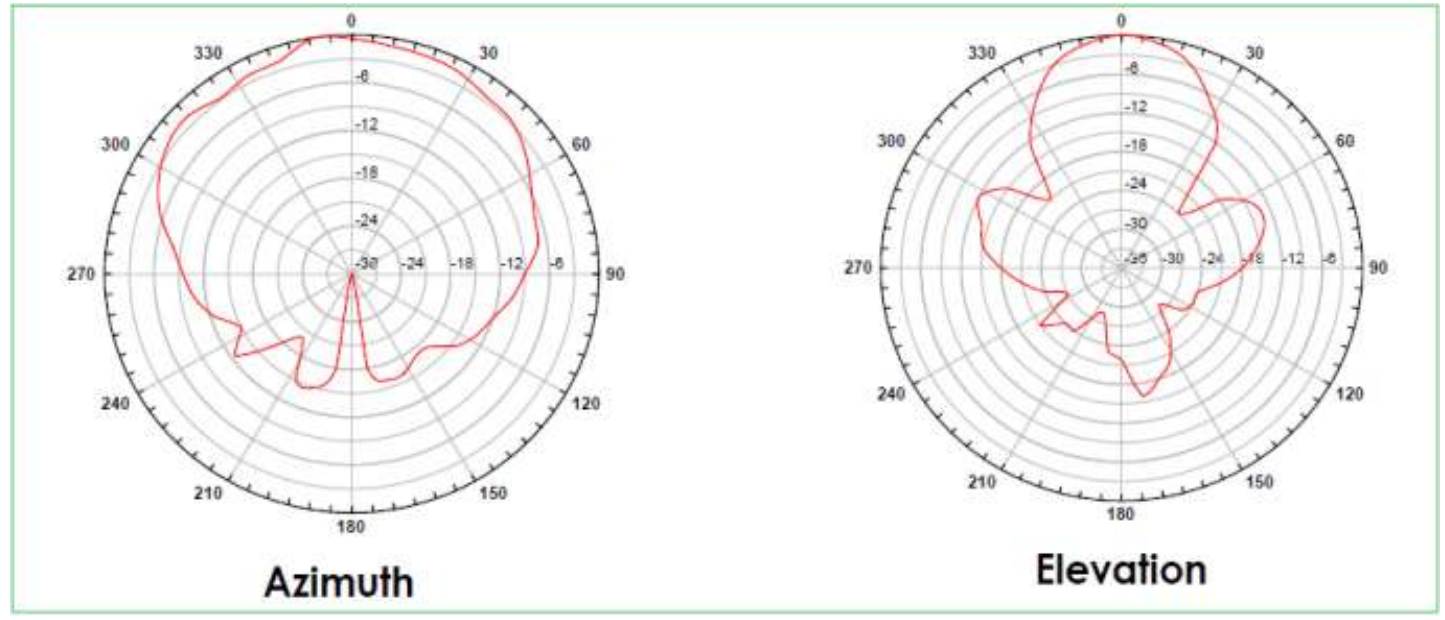

Chart - 1: The Radiation Pattern of HB 100

The module is to be mounted with the antenna patches facing to the desired detection zone. The radiation patterns of the antenna and their half power beam width (HPBW) are in chart -1 .

HB 100 module output voltage is very less it is need to be amplified in order to be detectable by the Arduino Uno. The figure 3 shows the amplifier circuit of the HB 100 radar module and its connection with the Arduino Uno.

This amplifier circuit consists of LM 324 amplifier to amplify the signal of the HB 100 module.

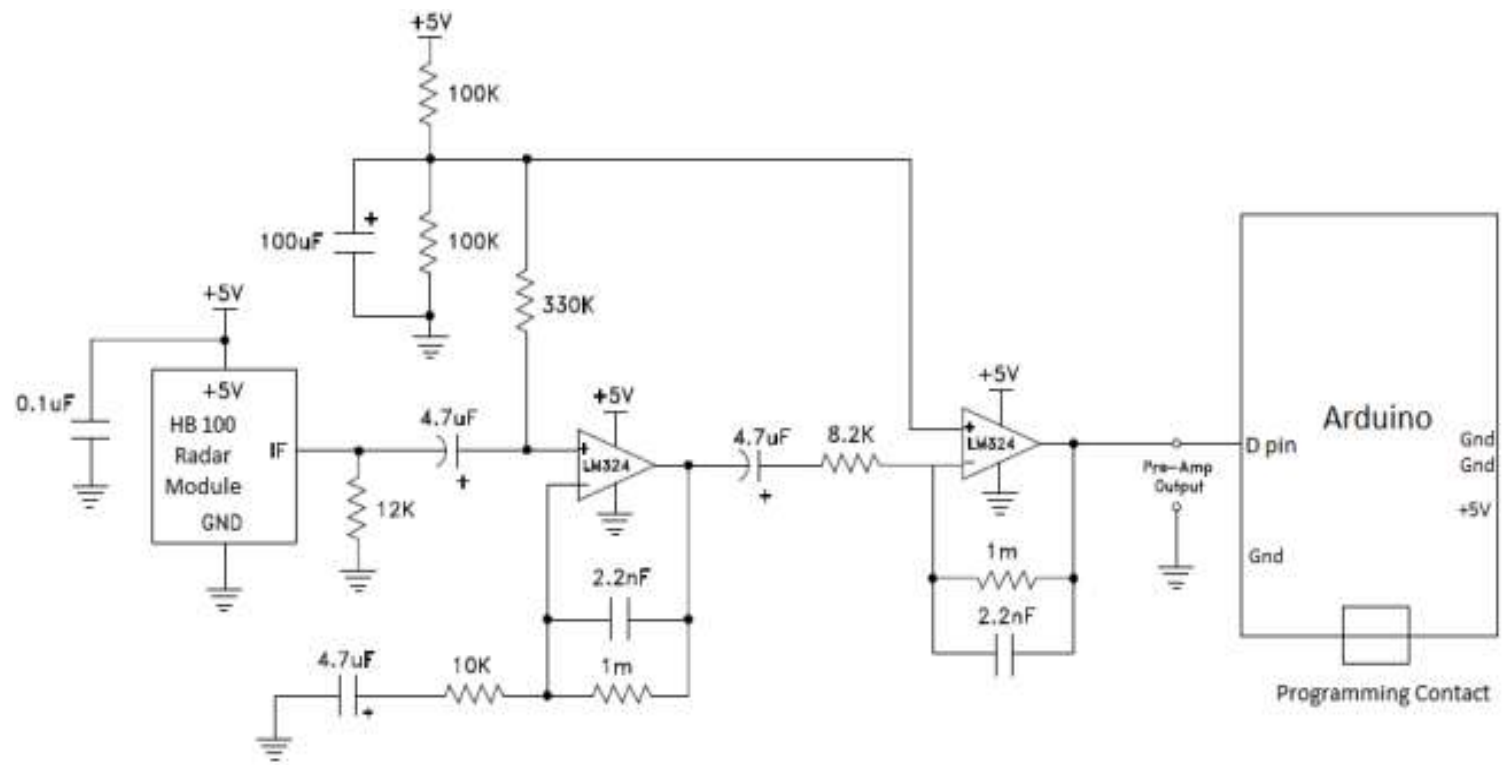

Fig 3: Amplifier Circuit for the HB 100 


\subsection{RCWL Human Body Induction Switch Module}

RCWL-0516 microwave radar sensor module Human body induction switch module intelligent sensor.

RCWL-0516 is a Doppler radar technology, specializedin the detection of moving objects in the microwave induction module.The module has the characteristics of high sensitivity, high induction distance, high reliability, large induction angle, wide power supply voltage range, etc. it is widely used in various kinds of human body induction lighting and alarm and so on. RCWL-0516 microwave radar sensor switch module human body induction module intelligent induction detectorhassensing distance of about 510 meters and the detection is not affected by the surroundings and it has better performance than the IR sensor.

The pin details of RCWL-0516 are as below:

- 3V3: 3.3V regulated output

- GND:Ground

- OUT Trigger: $3.3 \mathrm{~V}$ if motion detected

- VIN:4 - 28V supply voltage

- CDS:Enable control chip
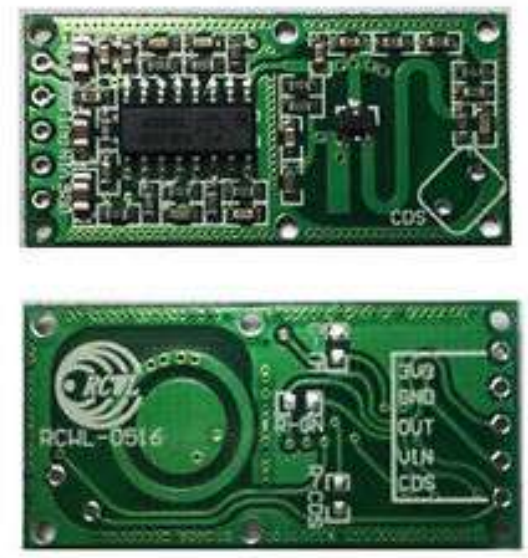

Fig 4: RCWL-0516 Human body Induction Switch Module design

\subsection{Arduino Uno}

Arduino Uno is a microcontroller board based on the ATmega328.It has 20 digital input/output pins(of which 6 can be used as PWM outputs and 6 analog inputs), a USB connection, a power jack, an ICSP header and a reset button. It contains everything needed to support the microcontroller; simply connect it to a computer with a USB cable or power it with AC-to-DC adapter or battery to get started.

The sensed data form RCWL-0516 and HB100 is given to Arduino. The instructions to the microcontroller are in such a way that if any movement of the human is detected in the range of the radar RCWL - 0516 immediately the breaks are being applied to reduce the speed or else if any other vehicle is being detected by the radar HB 100 if the vehicle is very close about a distance of few meters the brakes are being applied to reduce the vehicle speed. This operation of the vehicle is controlled by using a mobile application which communicates with the Arduino Uno with the help of a Bluetooth module HC -05 and the controls

Pin details of Arduino Uno:

Operating Voltage: $5 \mathrm{~V}$

Digital I/O Pins: 14

Analog Input Pins: 6 DC

Current per I/O Pin: $40 \mathrm{~mA}$ DC

Current for 3.3V Pin: $50 \mathrm{~mA}$

Flash Memory:32 KB

SRAM: $2 \mathrm{~KB}$

EEPROM: $1 \mathrm{~KB}$

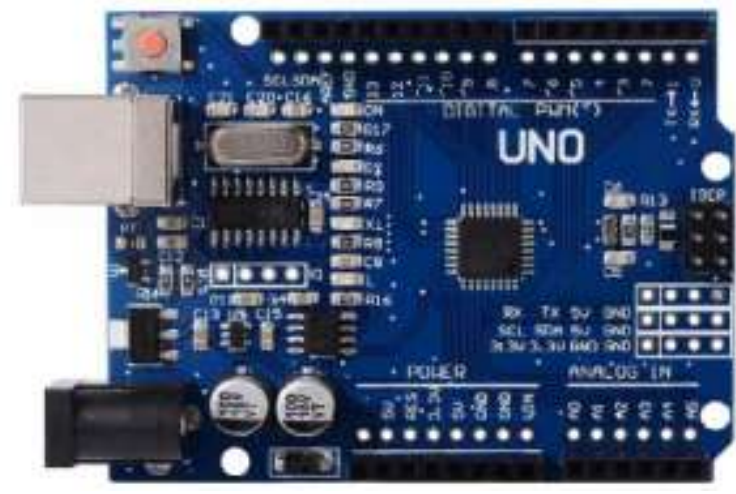

Fig 5: Arduino Uno

\subsection{Power Supply}

$\mathrm{V}_{\mathrm{IN}}$ the input voltage to the Arduino board when it's using an external power source (as opposed to 5 volts from the USB connection or other regulated powersource). To power up ArduinoUSB cable connected to a battery or power it with AC-to-DC adapter. Supplying voltage via the $5 \mathrm{~V}$ or $3.3 \mathrm{~V}$ pins bypasses the regulator, and can damage the Arduino Uno.

\subsection{Breaking Control System}

A motor driver IC is connected in order to control vehicle and for controlling the breaking system.Motor driver act as an interface between Arduino and the motor's. The motor driver IC used in the vehicle design isL293D.

The L293D is a 16 pin IC, with eight pins, on each side, dedicated to the controlling of a motor. There are 2 INPUT pins, 2 OUTPUT pins and 1 ENABLE pin for each motor. L293D consist of two H-bridge. H-bridge is the simplest circuit for controlling a low current rated motor. 


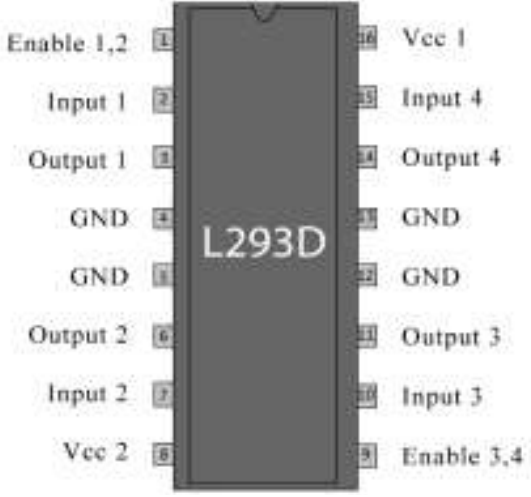

Fig 6: L293D Motor Driver IC

\section{PATCH ANTENNA DESIGN}

Using the ANSYS HFSS 15.0 we designed micro strip patch antenna. Patch Antenna is designed for resonant frequency of $23.5 \mathrm{GHz}$ with the dielectric constant of Rogers RO4350 material is $\epsilon_{\mathrm{r}}$ is 3.48 .

Rectangular patch antenna is developed for the transmission of RF signal of frequency $23.5 \mathrm{GHz}$ is used for the Vehicle Radar. This antenna is used for the transmitting the signal to the target and the reflected signal is collected by the antenna from the target.

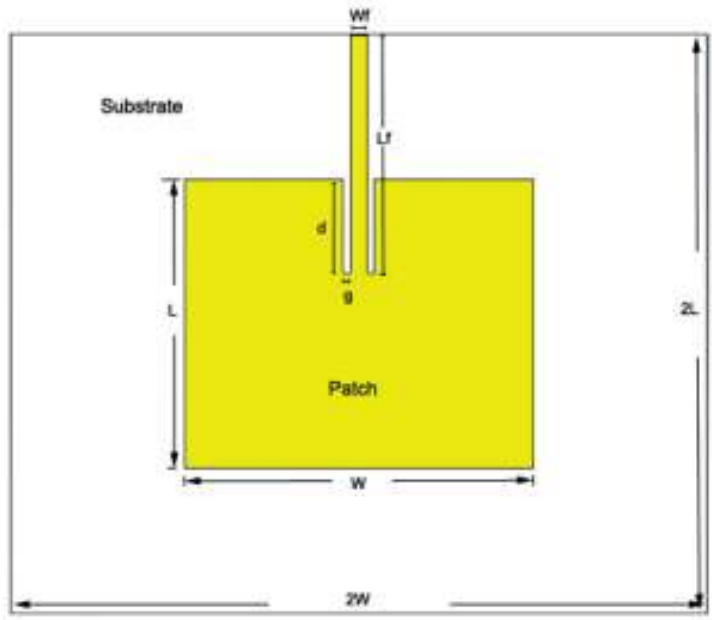

Fig 7: Micro strip patch antenna of resonant frequency of $23.5 \mathrm{GHz}$

The dimensions of the rectangular patch antenna are given by

Width of the patch $(\mathrm{W})=4.5 \mathrm{~mm}$

Length of the patch $(\mathrm{L})=3.13 \mathrm{~mm}$

Width of the micro strip line $(\mathrm{Wf})=0.1 \mathrm{~mm}$ Length of the micro strip line $(\mathrm{Lf})=1.81 \mathrm{~mm}$ Distance of gap $(\mathrm{g})=0.1$ $\mathrm{mm}$

Inset feed distance $(\mathrm{d})=0.75 \mathrm{~mm}$

Height of substrate $(\mathrm{h})=0.25 \mathrm{~mm}$

Thickness of patch $(\mathrm{t})=12 \mu \mathrm{m}$

\section{Micro strip patch antenna parameters are:}

\section{S - Parameter Plot}

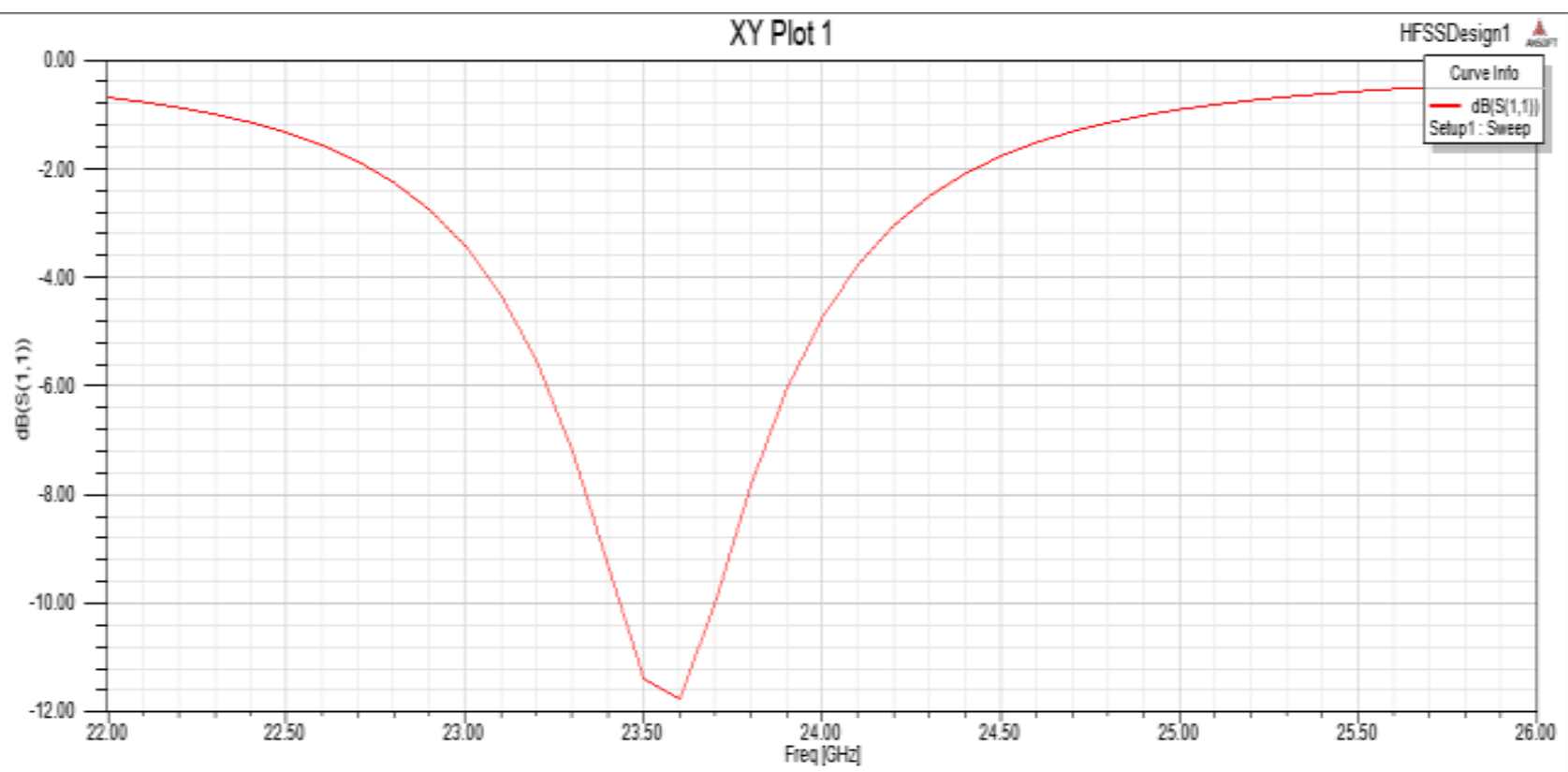

Chart -2: $\mathrm{S}$ - parameter plot of the rectangular micro strip patch antenna

From this plot we can infer that resonant frequency of the antenna is occurred at the frequency of $23.5 \mathrm{GHz}$ which is observed from the chart-2. 


\section{Gain of the Micro Strip Antenna}
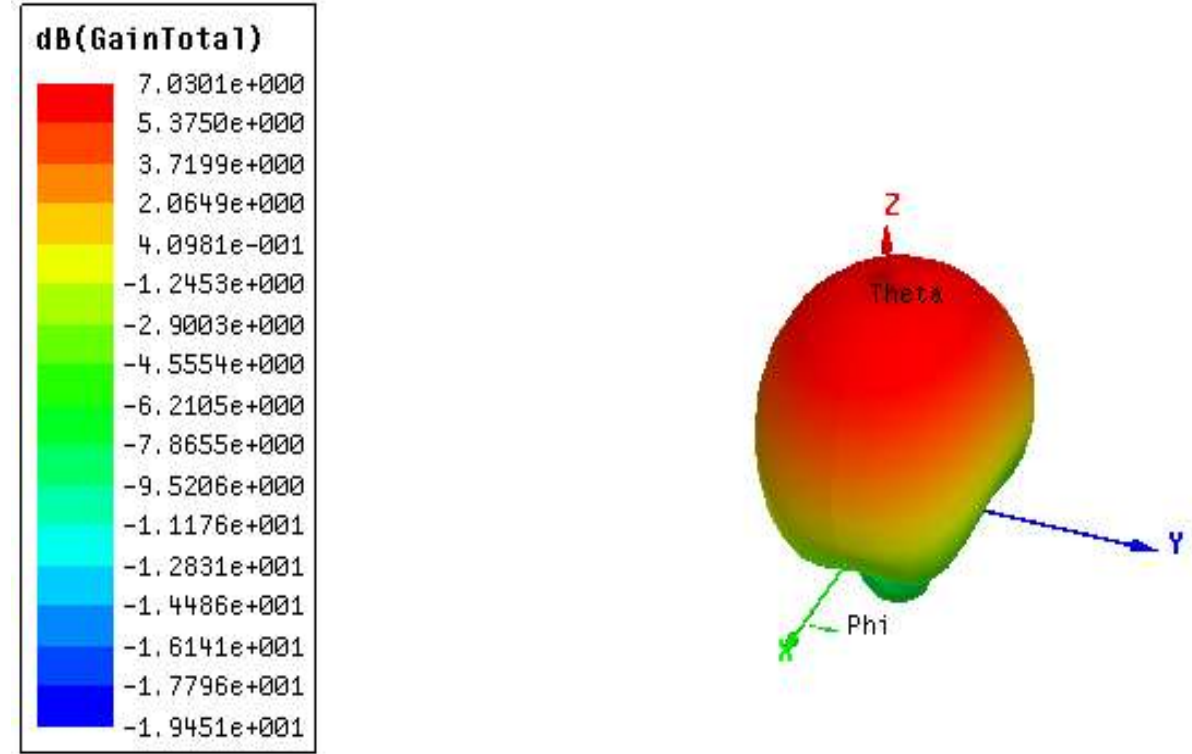

Chart - 3: Gain of the rectangular micro strip patch antenna

There are no side lobes and the antenna is highly directional from the radiation pattern in the above chart -3 .

\section{SIMULATION OF THE ASDCAR}

Using LAB View 2014 we have developed anAutomobile Speed Detection and Collision Avoidance Radar which works on the principle of the Doppler frequency shift.

Doppler Equation that we have considered is

$$
\mathrm{f}_{\mathrm{r}}=\mathrm{f}_{\mathrm{s}}\left[\left(\mathrm{c}-\mathrm{V}_{\mathrm{T}}\right) /\left(\mathrm{c}-\mathrm{V}_{\mathrm{S}}\right)\right]
$$

Where,

- $\mathrm{f}_{\mathrm{r}}=$ Received frequency

- $\quad \mathrm{f}_{\mathrm{s}}=$ Source frequency

- $\quad \mathrm{c}=$ Velocity of the EM wave (or) Light

- $\quad \mathrm{V}_{\mathrm{T}}=$ Target Velocity

- $\quad \mathrm{V}_{\mathrm{S}}=$ Source Velocity

Equation of Velocity $\left(\mathrm{V}_{\mathrm{T}}\right)=\mathrm{c}-\left(\mathrm{c}-\mathrm{V}_{\mathrm{S}}\right) \mathrm{f}_{\mathrm{r}} / \mathrm{f}_{\mathrm{S}}$

We know that distance between the target and source is given by $(\mathrm{D})=\left(\mathrm{V}_{\mathrm{T}}-\mathrm{V}_{\mathrm{S}}\right) \mathrm{t}$

Where, $\mathrm{t}=$ time in sec

Using this equation we have designed the Lab VIEW based Simulation of our Project the front panel and block diagram is designed.In this process we have developed a simulation to launch Anti-Lock Breaking System if the distance gap is less than or equal to $1 \mathrm{~m}$. The breaking system is automatically disabled once the gap is being increased. This system operation is simulated and the results were analyzed by providing different frequencies and by using the simulation result we have come to the conclusion that 23.5 $\mathrm{GHz}$ frequency is most suitable for the application of the Automobile Speed Detection and Collision Avoidance Radar. 


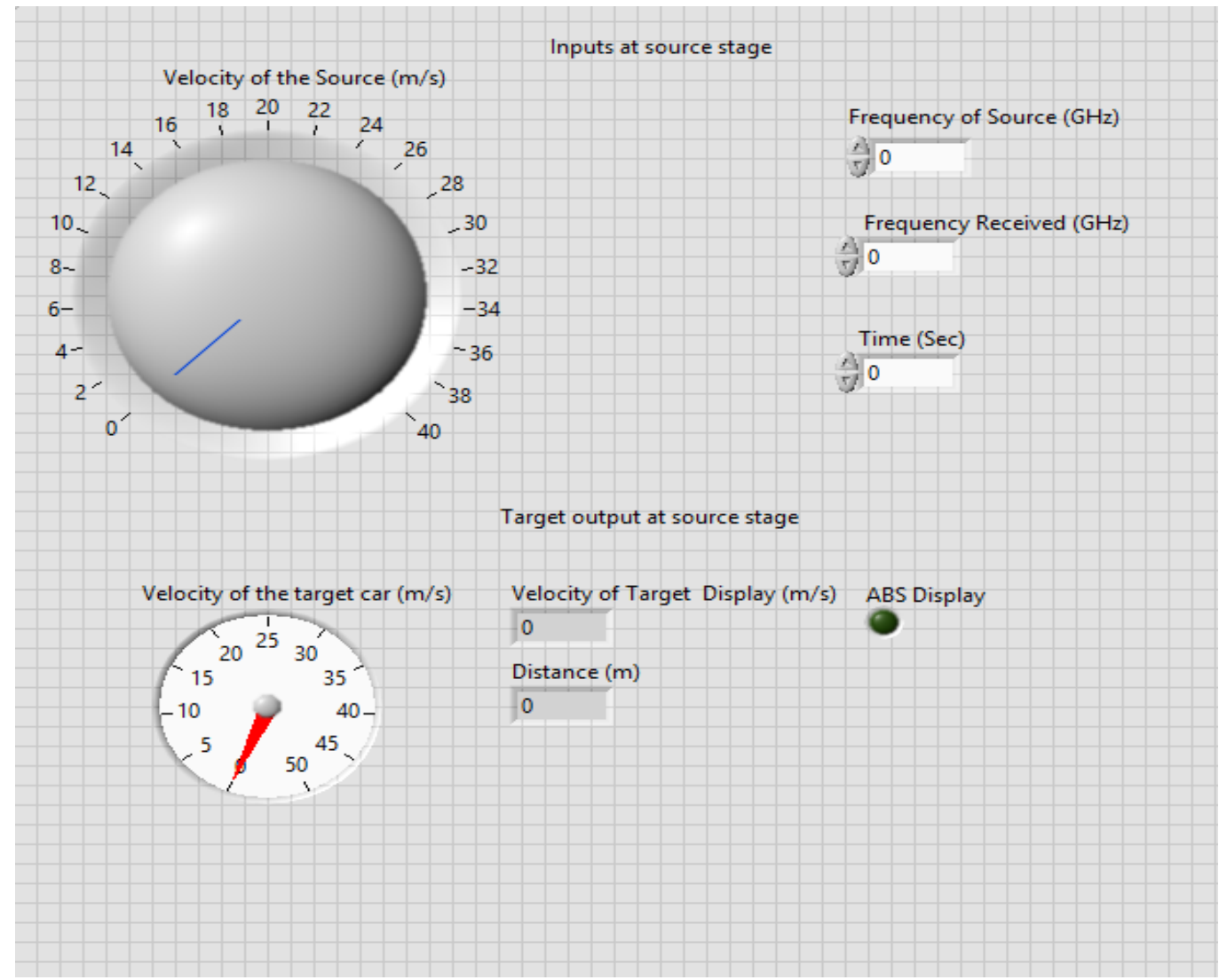

Fig 7: Front Panel of Automobile Speed Detection and Collision Avoidance Radar

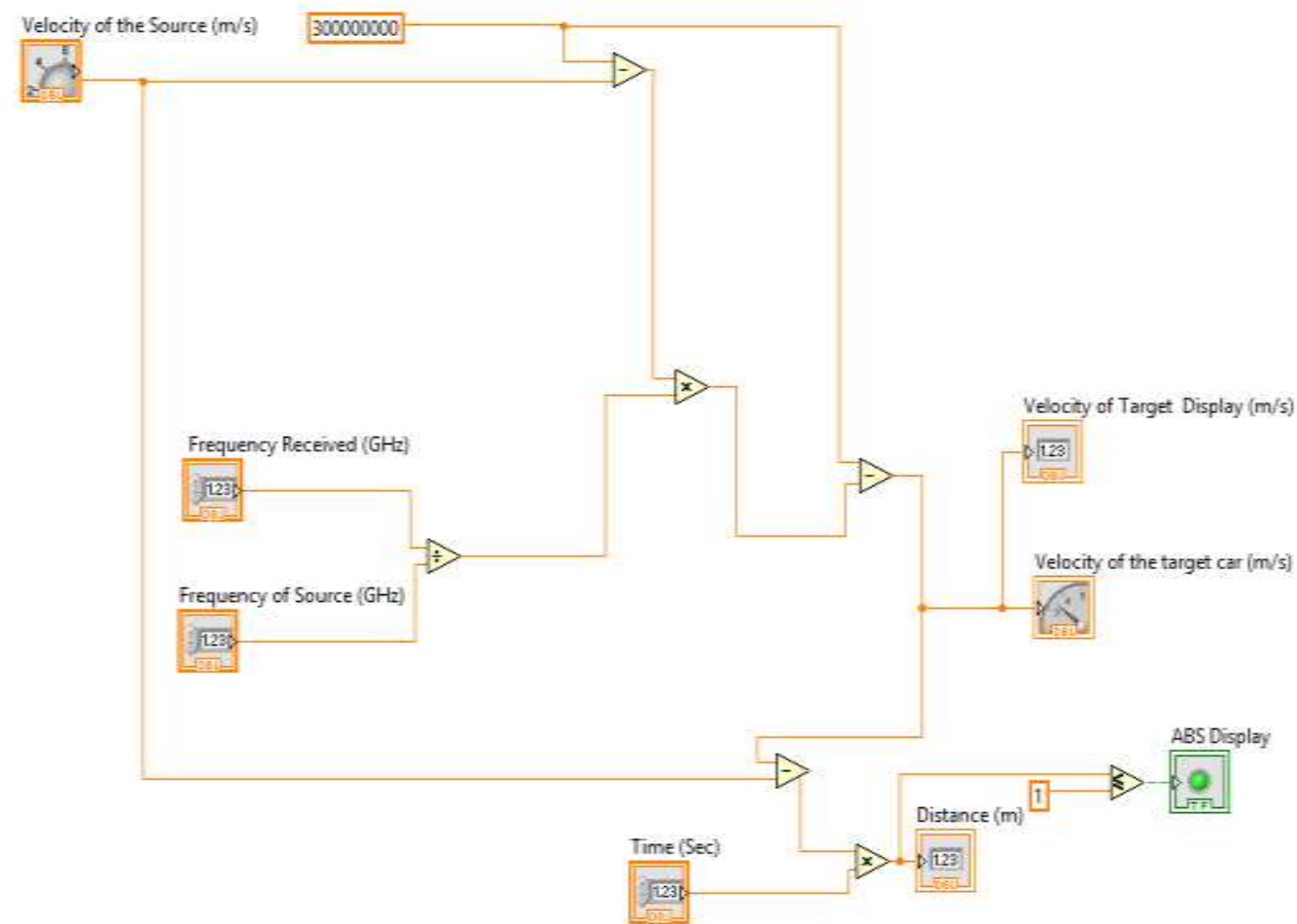

Fig 8: Block Diagram of Automobile Speed Detection and Collision Avoidance Radar 


\section{APPLICATIONS}

The major application of this ASDCAR is the Vehicle Safety Equipment. It can detect the speed and exact distance of the car ahead or behind. It can provide the speed to be maintained so that you can prevent collision with other vehicles. Anti - lock braking system can be linked to it to avoid collision when thedegree of closeness is increased.It helps to detect the vehicles presence in the blind spot region of the driver.It can detect any obstacle coming toward the vehicle i.e. humans and animals while crossing the road. It can be used by the Police for the speed detection of the vehicle while travelling in the care without using the conventional Doppler radar for the detection.

\section{CONCLUSION}

From the results of the design of the antenna design and the simulation of the Automobile Speed Detection and Collision Avoidance Radarin Lab View we have found that the radar can operate in the frequency range of the $23.5 \mathrm{GHz}$ and we have tested the prototype of the radar using the HB 100 and RCWL - 0156 modules and it is found to be operational and results from the short distance prototype testing is satisfactory and we are planning to develop the prototype of the $23.5 \mathrm{GHz}$ frequency range Automobile Speed Detection and Collision Avoidance Radar.

\section{ACKNOWLEDGMENT}

This research was supported by Prof. B. Sada Siva Rao who provided insight and expertise that greatly assisted the research. We would like to thank our college for providing the proper platform for the research and for the technical support for the research work.

\section{REFERENCES}

[1] Automotive Radars a Review of Signal processing Techniques by SujeetPatole, Murat Torlak, Dan Wang, and Murtaza Ali in IEEE SIGNAL PROCESSING MAGZINE, MARCH 2017

[2] $\mathrm{Ka}$ - Band Doppler Radar in Robust and Precise Cardiopulmonary Remote Sensing by Seyed Mohammad Ali TayaranianHosseini andHamidrezaAmindavar IEEE MAGZINE 2017.

[3] $77 \mathrm{GHz}$ Radar Sensor for Car Application by Hermann Rohling Ernst Lissel IEEE INTERNATIONAL RADAR CONFERENCE IN 1995.

[4] Automotive Radar by DALE M. GRIMES PROCEEDINGS OF THE IEEE, JUNE 1974.

[5] SPEED CONTROLING USING DISTANCE DETECTOR IN MOVING VEHICLE Amit Kumar Dimha, Vijay Kumar and John Singh. K, International Journal Of Engineering And Computer Science ISSN:2319-7242 Volume 2 Issue 5 May, 2013 Page No. 1453-1456.

[6] A Multi frequency Radar System for Detecting Humans and Characterizing Human Activities for Short-Range Through-Wall and Long-Range Foliage Penetration Applications by Ram M. Narayanan, Sonny Smith, and Kyle A. Gallagher in the Hindawi Publishing Corporation International Journal of Microwave Science and Technology Volume 2014, Article ID 958905, 21 page.

\section{BIOGRAPHIES}

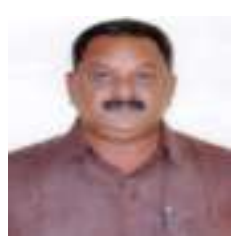

Prof. B. Sada Siva Rao B.E., M.E., PhDProfessor and H.O.D. of Department of Electronics and Communication Engineering GayatriVidyaParishad College for Degree and PG Courses School of Engineering

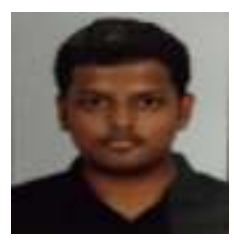

P. PydiSaiCharan Student of Bachelor of Engineering (BE) Department of Electronics and Communication Engineering GayatriVidyaParishad College for Degree and PG Courses School of Engineering

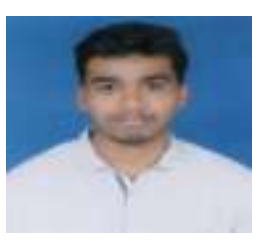

Ambati Nikhil Student of Bachelor of Engineering (BE) Department of Electronics and Communication Engineering GayatriVidyaParishad College for Degree and PG Courses School of Engineering

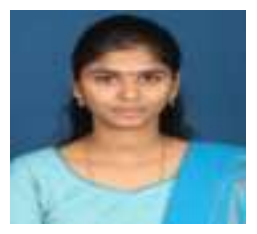

ItlaSnehaMounika Student of Bachelor of Engineering (BE) Department of Electronics and Communication Engineering GayatriVidyaParishad College for Degree and PG Courses School of Engineering

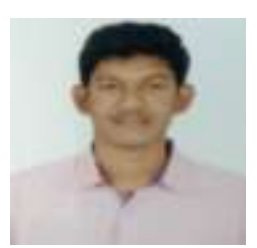

Engineering

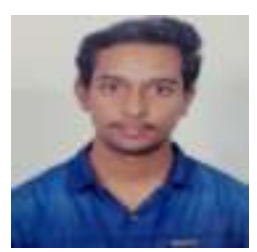

GantaHaswanth Kumar Student of Bachelor of Engineering (BE) Department of Electronics and Communication Engineering GayatriVidyaParishad College for Degree and PG Courses School of

V. ShyamSandeep Student of Bachelor of Engineering (BE) Department of Electronics and Communication Engineering GayatriVidyaParishad College for Degree and PG Courses School of Engineering 
MahboobBaig Student of Bachelor of Engineering (BE) Department of Electronics and Communication Engineering GayatriVidyaParishad College for Degree and PG Courses School of Engineering 